\title{
International consensus statement on injury surveillance in cricket: a 2016 update
}

\author{
John W Orchard ${ }_{1}^{1,2}$ Craig Ranson, ${ }^{3}$ Benita Olivier, ${ }^{4}$ Mandeep Dhillon, ${ }^{5}$ Janine Gray, ${ }_{1}^{6,7}$ \\ Ben Langley, ${ }^{8}$ Akshai Mansingh, ${ }^{9}$ Isabel S Moore, ${ }^{3}$ Ian Murphy, ${ }^{10}$ Jon Patricios, ${ }^{11,12}$ \\ Thiagarajan Alwar, ${ }^{13}$ Christopher J Clark, ${ }^{14}$ Brett Harrop, ${ }^{15}$ Hussain I Khan, ${ }^{16}$ \\ Alex Kountouris, ${ }^{2}$ Mairi Macphail ${ }^{17}$ Stephen Mount, ${ }_{1}^{18}$ Anesu Mupotaringa, ${ }_{1}^{19}$ \\ David Newman, ${ }^{8}$ Kieran O'Reilly, ${ }^{20}$ Nicholas Peirce, ${ }^{8,21}$ Sohail Saleem, ${ }^{16}$ \\ Dayle Shackel, ${ }^{10}$ Richard Stretch, ${ }^{22}$ Caroline F Finch ${ }^{23}$
}

- Additional material is published online only. To view please visit the journal online (http://dx.doi.org/10.1136/ bjsports-2016-096125)

For numbered affiliations see end of article.

\section{Correspondence to} Dr John Orchard, Sports Medicine at Sydney University, Cnr Western Ave \& Physics Rd University of Sydney, Sydney, NSW 2006, Australia; johnworchard@gmail.com

Accepted 12 May 2016 Published Online First 8 June 2016
CrossMark

To cite: Orchard JW,
Ranson C, Olivier B, et al. Br
J Sports Med
2016;50:1245-1251.

To cite: Orchard JW, J Sports Med

2016:50:1245-1251.

\section{ABSTRACT}

Cricket was the first sport to publish recommended methods for injury surveillance in 2005. Since then, there have been changes to the nature of both cricket and injury surveillance. Researchers representing the major cricket playing nations met to propose changes to the previous recommendations, with an agreed voting block of 14 . It was decided that 10 of 14 votes $(70 \%)$ were required to add a new definition element and 11 of $14(80 \%)$ were required to amend a previous definition. In addition to the previously agreed 'Match time-loss' injury, definitions of 'General time-loss', 'Medical presentation', 'Player-reported' and 'Imagingabnormality' injuries are now provided. Further, new injury incidence units of match injuries per 1000 player days, and annual injuries per 100 players per year are recommended. There was a shift towards recommending a greater number of possible definitions, due to differing contexts and foci of cricket research (eg, professional vs amateur; injury surveillance systems vs specific injury category studies). It is recommended that researchers use and report as many of the definitions as possible to assist both comparisons between studies within cricket and with those from other sports.

\section{INTRODUCTION}

In 2005, cricket was the first sport to publish recommended methods for injury surveillance. ${ }^{1}$ This has given rise to cricket injury surveillance publications that have employed relatively consistent methodologies. ${ }^{2-6}$ However, there has not been universal uptake of the original definitions due to issues such as the exclusion of non-time-loss injuries from the main injury definition, ${ }^{6-8}$ and updated definitions that have been introduced by some authors in response to the rise of T20 cricket. ${ }^{4}$ T20 cricket was a very minor part of the cricket calendar in 2005 and the original definitions were focused on longer forms of the game, but in the decade since, T20 cricket has emerged as an extremely prominent format of the game. For these reasons, during a series of meetings in 2014 and 2015 , cricket injury researchers agreed to produce a revised injury surveillance consensus statement (to be called the 2016 update).

The original primary definition of a cricket injury was a 'match time-loss' definition (table 1). ${ }^{1}$ It was also noted that some cricket studies and surveillance systems might use a broader definition of injury such as a 'medical presentation' (table 1). Football (soccer) ${ }^{9}$ and rugby union ${ }^{10}$ followed cricket in publishing consensus statements, choosing to recommend three levels of definition, based on 'all physical complaints', 'medical presentations' and 'time-loss' (table 1). There has been debate about whether narrow/reliable definitions should be used in consensus statements ${ }^{11}$ or whether broader definitions are needed. ${ }^{7}$ It has also been proposed that tissue pathology/damage, which may be present even before an athlete becomes symptomatic, is the ultimate precursor to 'all injuries' (table 1). ${ }^{8} 12$

Since the definition of an injury may be different depending on the context of the study or research aim, an attempt by a consensus statement to encourage all research projects to use the same definitions is not necessarily appropriate. ${ }^{13}$ Therefore, this new consensus encourages research and injury surveillance projects to:

1. Be very clear on the exact methods that have been used, so that studies can be clearly understood and reproducible. Rather than only giving a brief statement that 'consensus methods were used', a description of the consensus methods employed should be provided;

2. When possible, multiple methods of presenting injury rates should be included so that other studies and systematic reviews can compare and compile different studies.

\section{METHODS}

The process of updating definitions was initiated and formatted at several face-to-face meetings:

1. On 13 April 2014 at the 4th IOC World Conference on Prevention of Injury and Illness in Sport in Monaco, attended by CF, ISM, IM, JO, JP, CR and RS;

2. On 26 March 2015 at the 5th World Congress on Science and Medicine in Cricket, in Sydney, attended by MD, CF, JG, BL, AM, BO, JO, CR and TA;

3. On 28 August 2015 in Cardiff between the two senior authors JO and CR.

Sadly, one of our proposed consensus group and the first author to publish multiple studies ${ }^{14-17}$ on cricket injury surveillance, RS, died after a short illness in late 2014. It was decided unanimously to include him posthumously in this consensus publication. 
Table 1 Types of definition of an injury possible in surveillance systems $^{12} 13$

\begin{tabular}{llll}
\hline Definition type & Scope & Reproducibility & Injury rates \\
\hline All tissue pathology & Most broad & Least reliable & Highest \\
All symptoms & Broader & Less reliable & Higher \\
All medical presentations & Moderate & Moderate & Moderate \\
Time-loss injuries & Narrower & More reliable & Lower \\
Match time-loss injuries & Most narrow & Most reliable & Lowest \\
\hline
\end{tabular}

The group determined by negotiation and eventual agreement that the following methods would be used as the basis for this new consensus statement:

1. That the previous consensus recommendations form the basis for this new statement, with the default definition for any component being the previous statement;

2. That changes and additions should be determined by a nomination process with a vote made on whether to accept or reject changes;

3. That voting for changes and additions would be primarily on the basis of country representation (one vote per International Cricket Council (ICC) member nation) with two additional voting blocks allowed outside the ICC members to represent; the ICC itself, community level (nonprofessional and age-group) cricket researchers;

4. That amending an item from previous recommendations required 11 of $14(\sim 80 \%)$ of the new group to agree to any proposed change;

5. To add a new item required a slightly lower threshold of 10 of $14(\sim 70 \%)$ of the new group to agree.

It was agreed that there should be 14 votes, shared among the 23 voting authors, as follows (voting authors in italics):

ICC (CR, IM), Community (non-elite) cricket (JP, DN), Australia (JO, AK), England (BL, NP), India (MD, AT), New Zealand (DS, IM), Pakistan (HIK, SS), South Africa (JG, BO), West Indies (AM, CJC), Bangladesh $(B H)$, Sri Lanka $(S M)$, Zimbabwe $(A M)$, Ireland (KO’R), Scotland $(M M)$.

$\mathrm{CF}$, who was a key leader of the consensus process, temporarily withdrew from the process during voting for personal reasons. She was re-included as a non-voting author after the voting process had been concluded.

There was no funding provided for a stand-alone meeting for all authors to attend, so the negotiations and voting processes were carried out with the entire group via email (to include representatives from countries who could not attend any of the meetings). The time period of April-September 2015 was allocated to nominate additions and changes to the first author for consideration. All nominated additions and changes were circulated to the group on 17 October 2015, with a time period of 3 months given for representatives to discuss the changes among themselves and for the voting representatives to indicate their decision to the first author via email.

A subgroup of JO, CR, BO and CF (a non-voting author) managed the revision process.

\section{RESULTS}

Where no reference to previous methods is made in these results, the methods previously published are retained. The following definition elements have been changed or recommended for inclusion by this consensus group (for a summary of all definitions, refer to online supplementary appendix I and figure 1):

\section{Injury definitions}

'Match time-loss' injuries

A 'significant' cricket injury in 2005 was defined as:

Any injury or other medical condition that either: (1) prevents a player from being fully available for selection for a major match or (2) during a major match, causes a player to be unable to bat, bowl or keep wicket when required by either the rules or the team's captain.

This definition is retained, but not as the only recommended definition of a cricket injury. Although previously referred to as a 'significant' injury, the preferred new term is 'match time-loss' injury.

For this category (and all other categories below), the definition is inclusive of illnesses, so that the term 'injury' refers to injury or illness.

With respect to notes on the definition for match time-loss injury, a domestic, franchise or international T20 match is now to be considered a major match.

It is noted that mention of 'batting with a runner' in the previous definition terms has been removed as runners are no longer allowed in major matches.

A further note of clarification is that, on the basis of the definition of causing an actual match to be missed, a player can only be suffering from one 'match time-loss' injury at any given time. For example, if a player suffers a concussion and fractured nose in a single incident and misses match playing time, only the more severe of the two (as determined by the medical staff) would count as a 'match time-loss' injury. With respect to other definitions provided later, both of these could be considered as injuries.

It should also be noted that when surveillance is being performed at community or amateur level, if a 'match time-loss' definition is used, then a major match will need to be defined.

Alternate injury definitions are optional in this new consensus statement and injury surveillance systems are encouraged to report injury rates in multiple formats. Since multiple definitions are permitted, studies which use 'consensus methods' need to make clear in their methods exactly which definitions were used.

\section{'General time-loss' injuries}

It was unanimously agreed to include an alternate injury definition of 'general time-loss' injuries. A general time-loss injury is any injury (or illness) that results in a player being considered unavailable for match-play, irrespective of whether a match or training was actually scheduled. The difference between a general time-loss injury and a match time-loss injury is that the latter only considers injuries that cause the player to be unavailable on scheduled match days, as opposed to at all times for general time-loss injuries, for example, training days, days off, off-season.

Three other injury definitions have been added to the new consensus statement.

\section{'Medical attention' injuries}

A medical attention injury is 'any health-related condition that required medical (or medical staff) attention and had the potential to affect cricket training or playing'. It therefore includes time-loss and non-time-loss injuries.

\section{'Player-reported' injuries}

A player-reported injury is any condition which was considered to represent an injury by the player (or parent/teacher on the 


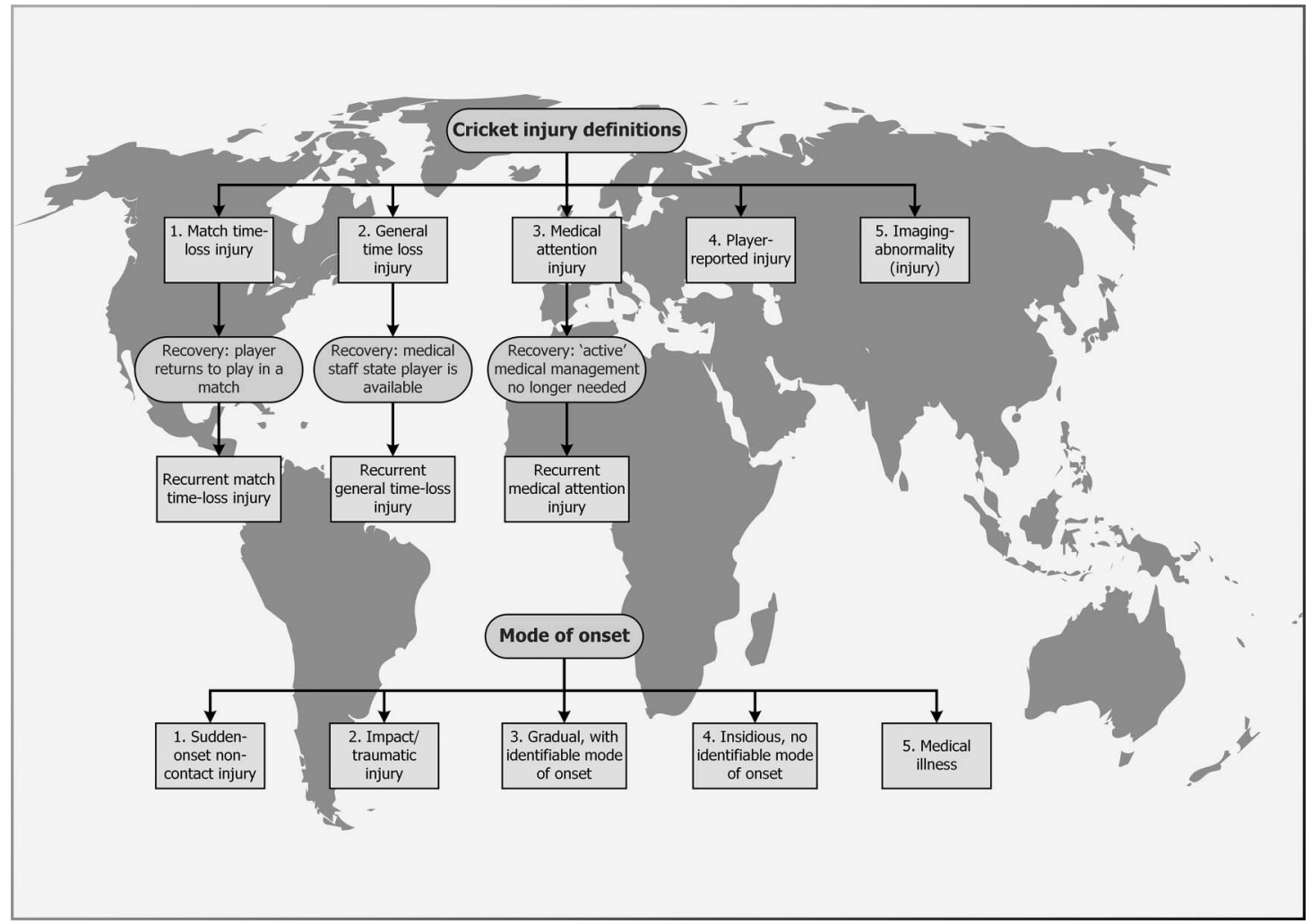

Figure 1 Summary of key injury definitions.

player's behalf, with respect to junior players in teams without medical staff). This definition is highly subjective and reliant on player engagement with injury surveillance. It is recommended (only) for use in surveillance of community cricket where medical staff are not necessarily in attendance. When using this definition, only regional diagnoses should be used (eg, knee, shoulder and back) and broad onset of injury (eg, batting, bowling and fielding) rather than with additional details, as players do not necessarily have the expertise to make accurate diagnoses.

\section{'Imaging-abnormality' injuries}

An imaging-abnormality (injury) is any condition which gives rise to abnormal findings on specific medical imaging. This definition is not recommended for general injury surveillance. It should only be used in studies that examine a specific body part or cricket injury type (eg, lumbar bone stress injuries) in conjunction with other (clinical) definitions. Only non-invasive and non-radiating imaging (eg, ultrasound, MRI) should be used to assess tissue integrity in an asymptomatic player (ie, when peformed for research and not for clinical management purposes). Any use of this type of injury definition must recognise that there is a high prevalence of abnormal imaging findings in otherwise clinically normal athletes.

\section{Mode of onset}

We suggest differentiating between the following different modes of onset:

1. Sudden-onset non-contact injury (eg, ankle sprain during the bowling run-up);

2. Impact/traumatic injury (blow or contact) (eg, fractured rib due to collision with another player);

3. Gradual onset associated with bowling/running/throwing/ batting practice/weight training (eg, low back pain with gradual onset which is experienced during and aggravated by the fast bowling action);

4. Insidious (gradual and no identifiable mode of onset) (eg, posterior thigh pain which started with no identifiable cause);

5. Medical illness (eg, the flu, gastroenteritis).

The mechanism of injury should be described as this will assist in an accurate mode of injury classification. It is furthermore important to specify whether the injury was sustained outside cricket as these may still affect cricket participation.

\section{Definitions of injury recovery and recurrence}

The definition of injury recurrence and injury recovery will depend on the specific injury definition employed. In general terms, a recurrent injury is one of the same type which reoccurs in the same season (surveillance year) after it has been defined as recovered.

\section{Match time-loss injuries}

As per the original recommendations, 'An injury is considered recovered once a player has returned to full (unrestricted) participation in at least one match (of any type or grade). A recurrent injury is one to the same side and body part, and of the same injury type, as an injury that previously qualified as an injury earlier in the same season, but which had recovered.' For a match time-loss injury, recovery is return to an actual match.

\section{General time-loss injuries}

Recovery from a general time-loss injury differs from recovery for a match time-loss injury, in that they have recovered once the medical staff consider that the player has returned to match availability, regardless of whether a match is scheduled for that day or not. 
Medical presentation injuries

Recovery from a medical presentation injury is determined by the injury no longer needing 'active' medical management (or, if the injury required ongoing medical management for the entire surveillance period, at the end of that surveillance period). Recurrence occurs when an injury that no longer required medical management re-entered this category in the same year.

It should be noted that some cricket injuries may never fully recover during a player's career. However, ongoing treatments aimed at maintaining, rather than improving a player's condition (such as strapping, manual therapy and exercises aimed at injury prevention), are not considered 'active' medical interventions. Moreover, the occurrence of some injuries may be related to previous injuries, even when they are not defined as recurrences or have fully resolved. ${ }^{18}$

\section{Injury incidence measures}

Calculation of injury incidence

Injury incidence analyses the number of new injuries (or new plus recurrent) occurring over a given time period, and should be measured in either or all of the following major formats.

\section{Match injury incidence}

Match incidence considers only those injuries occurring during major matches. This can be calculated in two types of unit (overall, with a time-based denominator and with a deliverybased denominator if considering batting or bowling injuries separately). A 'delivery' is the preferred term in cricket for a ball, although the two terms are interchangeable (analogous to a baseball pitch). Six deliveries make up one over, which is the other major unit of counting bowling.

The numerator should be the 'number of injuries', and can include either new injuries or total injuries (new plus recurrent). The denominator should either be stated as injuries per number of player hours, or number of player days, or both (1).

Calculation of batting and/or bowling match incidence can be made with a delivery-based denominator. The numerator should be the number of batting injuries and/or bowling injuries, and can include either new injuries or total injuries (new plus recurrent). The denominator for bowling match injuries should be deliveries (or overs) bowled. The denominator for batting match injuries should be deliveries faced. The preferred units being:

Match injuries from all phases (batting, bowling and fielding): injuries per 1000 player days (or matches for community levels of cricket where multiple days are not played); bowling injuries: injuries per 10000 deliveries bowled; batting injuries: injuries per 10000 deliveries faced. Bowling injuries could alternatively be converted into a unit of bowling injuries per 1000 overs bowled ( 1 over $=6$ deliveries $)$.

Match injury incidence is the preferred injury incidence measure to be used for tournaments where multiple teams play an intense period of matches in a specific time period.

Recommended new incidence unit: match injuries (new, recurrent or combined) per 1000 player days.

\section{Training injury incidence}

Training incidence can be separately measured from match incidence, although measuring exposure is extremely difficult, given the multiple phases of training (batting, bowling, fielding, general conditioning and resistance/strength training). The difficulty of subdividing training injuries for all phases (including exact calculation of exposure) is probably beyond even the most sophisticated current injury surveillance systems. However, specific measurement of bowling workload at training (number of deliveries bowled) is both recommended and now undertaken by many teams, so bowling injury incidence at matches and training can be measured. It is recommended to use the same units (and therefore count or calculate deliveries bowled, rather than overs bowled in training sessions, so as to be able to use the unit of bowling injuries per 10000 deliveries bowled).

\section{Seasonal and yearly injury incidence}

Seasonal incidence is defined as the number of defined injuries occurring per squad per season. This allows match injuries, training injuries and also gradual onset and insidious onset injuries to be combined in the one measurement. A squad in 2005 was defined as 25 players, and a season was defined as 60 days of scheduled match play. ${ }^{1}{ }^{4}$ 19-21 The recommended unit of measurement was injuries per squad per season, with a 'squad season' being defined as 1500 (25 multiplied by 60) player days. The use of an injury incidence measurement which combined match, training and gradual onset injuries is particularly useful when performing longitudinal surveillance, especially given studies which have reported high workloads leading to delayed rather than immediate increase in injury risk. ${ }^{22} 23$

However, the choice of a squad being 25 players and a season being 60 match days is no longer representative of international cricket in particular, which has evolved into a 9-12-month schedule for the major teams. Squad size and number of days could be adjusted to compare different scenarios. However, for simplicity and ease of comparative calculation, we now recommend using annual injury incidence rather than seasonal injury incidence. This considers a temporal exposure of 365 calendar days rather than 60 match days, and given this change, a study should report the actual number of match days for a squad/team in a given year. In terms of determining the recommended size of a squad, 100 players is also recommended for simplicity. In practice, no squad is as large as 100 players, but there is also no longer a 'typical' squad size. If 100 players are chosen, the unit of injuries per 100 players per year can easily be recalculated to any preferred squad size. The number of injuries per 100 players per year can also rapidly be converted (with a move of the decimal point) as number of injuries per player per year.

Recommended new incidence unit: annual injuries (new, recurrent or combined) per 100 players per year. Incidence can be reported as a rate with an accompanying 95\% CI. This should be always be carried out when surveying just a sample of the population (eg, a few of many teams in a competition).

\section{Injury prevalence measures}

Injury prevalence considers the average number of squad members not available for selection through injury or illness for a given time period, divided by the total number of squad members. Injury prevalence should be expressed as a percentage, representing the percentage of players missing through injury on average for that team for the season in question.

Injury prevalence as calculated in the 2005 recommendations should now be referred to as 'match injury prevalence'. It is calculated using the numerator of 'missed player games', with a denominator of number of games multiplied by the number of squad members. An injury prevalence figure of, say, 12\% indicates that for the matches played in the time period under survey, on average $12 \%$ of players were unavailable for matchplay because of injury or illness.

Injury prevalence can also be presented, also as a percentage of players unavailable, on the basis of general time-loss status taking into account daily status over a tournament or a 365-day period. This injury prevalence should be referred to as 'annual 
injury prevalence' when used for 365 days or 'general injury prevalence' if used, say, for a tournament. Match injury prevalence and annual injury prevalence will be related as the former is a subset of the latter. Match injury prevalence only takes into account status on days where major matches are actually scheduled, whereas annual injury prevalence includes the former plus training and off-days.

\section{Characterisation of player role and activity at the time of injury}

With respect to injury prevalence measures and yearly (or seasonal) injury incidence rates, a player should be categorised by his or her usual playing role as either a: batsman, fast (pace) bowler, slow (spin) bowler or wicketkeeper.

With respect to match injury incidence within the various phases of the game, a player should be categorised by the role they were undertaking at the time of injury, that is, batting, bowling or fielding. Therefore, a player who is usually a fast bowler is categorised as such when taking into account injury prevalence and yearly injury incidence; when considering the rate of bowling match injuries, this player is only considered as a bowler in the act of actually bowling. When batting, any injury sustained (even by a player who is considered a bowler) is a batting injury and when injured fielding one's own bowling, this injury is considered a fielding injury.

In the previous recommendations, a regular bowler was defined at the start of each season as a player who averaged more than five overs bowled in matches played during any of the previous two seasons. This criterion should be superseded, as a T20 bowler is only allowed to bowl a maximum of four overs per match. Bowlers, therefore, are now best defined as players who have bowled more than $10 \%$ of the overs bowled by their team in matches that they played in, for either of the two previous seasons ('either of two' qualifications included to capture an 'all-rounder' who may have played as a batsman only for a part of a single season due to injury). This $10 \%$ rule can also be applied to wicketkeepers (a wicketkeeper is a player who has kept wicket for more than $10 \%$ of overs that they have been on the field for, meaning that part-time wicketkeepers are defined generally as wicketkeepers rather than batsmen).

The position of 'all-rounder' is not generally recommended for surveillance purposes, as every bowler is required to bat at times and many batsmen can occasionally bowl. However, if it is to be used, the suggestion of using batting average as a cut-off (made in the previous recommendations) has been rendered unwieldy by the rise of T20 cricket. A preferred definition now is that an 'all-rounder' is a regular bowler (ie, someone who regularly bowls at least $10 \%$ of a team's overs) who, for the majority of the innings, bats in the top seven batting positions.

Bowlers should be stratified initially (and primarily) into fast (or pace) and slow (or spin). The categorisation between the two is usually clear-cut, with a key difference being that the wicketkeeper will always stand directly behind the stumps for slow/spin bowlers. Bowlers can be subcategorised, within the pace spectrum, as 'fast', 'fast-medium' and 'medium', and within the spin category as off/finger spin and leg/wrist spin. The player profiles listed by ESPN (Wisden) Cricinfo (http:// www.espncricinfo.com/) tend to be universally accepted.

\section{ADDITIONAL DEFINITIONS OF TIME FRAMES AND COHORTS \\ Definition of time-frames}

Although cricket has traditionally (in the temperate countries) been played for $\sim 6$ months of the year; at the international and professional level, the calendar now approaches 12 months of competition. At amateur levels, competition is typically held over shorter blocks, for example, 6 months. To account for this distinction, it is suggested to move from measures of 'seasonal' injuries to 'annual' injury rates.

A suggested start date for annual surveillance may be at the end of the southern hemisphere season or start of the Indian Premier League (IPL) competition (when many international teams have a 'window' in their schedule), although this should not be prescriptive. It remains sensible for some northern hemisphere teams to use the calendar year. Regardless of what start date is chosen, it should be included in the methods of injury surveillance research papers.

When conducting surveillance for periods less than a year, the following guidelines could be used:

1. The traditional 6-8-month cricket season could also report 'annual' injury rates with the notation that this may miss 'off-season' injuries. For (adult) players who only play one sport (ie, cricket), the injury rate for a cricket 'season' would almost equate to an 'annual' rate. For junior players who may play, say, one of the football codes in winter and cricket in summer, it would be important to note that a rate was a 'seasonal' incidence rate (not including injuries from the other sport) rather than an 'annual' incidence rate (which would include injuries sustained while playing other sports).

2. For a tournament that lasted for, say, 2 months, injury rates could theoretically be multiplied by six to give an 'annual' injury incidence based on the hypothetical situation of a similar tournament being indefinitely played six times per year. However, in reporting tournament injury incidence (eg, for a World Cup, or in a domestic T20 competition), it may be preferable to use match injury incidence rates rather than annual incidence rates.

\section{Definition of cohorts}

The cohort to be followed for a given team should be referred to as the 'squad'. A match-day team generally consists of 11 players (11 active players and the 12th man), whereas a squad for a team contains a varied number of players. The squad to be followed can consist of any number of players, which should be recorded for all times of surveillance (ie, for the entire year if annual surveillance is being undertaken).

A squad should be chosen at the start of the surveillance period, determined in advance. Therefore, injury rates will consider those players who become injured as well as those who avoid injury, and it is possible that a squad will contain injured players at the start of the surveillance period. A squad might consist of a team's designated list of contracted or registered players, or a group of players selected for a particular tournament. However, players may need to be added if they are chosen to play for the team from outside the initial squad. Similarly, players who are de-registered, or are dropped from a contracted professional or international squad, should be removed.

The definition of a squad member for a team may be varied, but an example is as follows:

1. Any player under contract to the team in question (or a 'registered' player at amateur level).

2. Any other player who plays in the team first XI (not including 12th man or a fielding substitute) or tours overseas with the team, from the time of his/her first game (or the first tour match) until the end of the cricket year or season (when a new round of contracts is awarded). 
For tournament injury surveillance, if the cohort is determined to be the selected squad for the tournament, it should be noted that there would be a strong bias towards injured players not being selected (although occasionally they are, in the hope that they may become fit early on during the tournament). Injury prevalence for tournaments with cohorts chosen in this fashion are likely to be lower than injury prevalence for contracted players on an annual basis, as the tournament cohort is biased against players suffering from pre-existing injuries (who make up a high proportion of the injured cohort when considering injury prevalence).

For additional information pertaining to injury surveillance systems, refer to online supplementary appendix II.

\section{DISCUSSION}

This consensus statement has evolved from the original recommendations of 2005. The evolution of this consensus statement is from being prescriptive ('all studies should use the following methods') to descriptive ('all studies should conduct the most complete injury surveillance possible and clearly describe the methodology'), emulating statements such as PRISMA ${ }^{24}$ and STROBE. ${ }^{25}$

A greater motivation for an updated consensus statement is to lead to consistency within the sport of cricket as well as between sports, using definitions that may be compared to those used in other sports. There has also been a greater appreciation that it is impossible and unwise to try to formulate a fixed set of definitions to suit all types of studies within a single sport. Given the concession that definitions will therefore need to vary, the focus of this consensus statement has moved to clarity in choosing and reporting methods used.

The difficulty in providing a single injury definition that will suit all purposes with respect to a sport or injury surveillance system within a sport has been discussed. ${ }^{12} 13$ To illustrate this argument in a cricket context, consider how shoulder injuries might best be defined in the following circumstances:

1. To determine whether certain countries/teams or years had higher rates of shoulder injuries than others, a 'time-loss' injury definition may be preferable, as it is the most easily captured, objective and reliable indicator of a significant injury;

2. To determine shoulder injury rates during a cricket tournament, a 'medical presentation' injury definition may be preferable, as all teams will have medical and/or physiotherapy staff who could hopefully keep consistent data over a limited time period during a tournament;

3. To determine shoulder injury rates in amateur cricketers, a 'player-report' definition based on physical symptoms may be preferable, as amateurs may or may not have presented to any medical staff with their symptoms but should be able to recall symptoms that have occurred within a reasonable time period, for example, the past year;

4. To study the development of pathology in the shoulder and its relationship to symptom development in cricketers, a study using an 'abnormal imaging' injury definition, based on ultrasound scan or MRI findings performed on cricketers without shoulder symptoms, could be employed.

Although time-loss definitions are preferable to medical attention injuries in many circumstances, there is one emerging injury category of importance where a medical attention definition is preferable: concussion and head impact injuries. Given the long-term concerns relating to concussion, it is recommended that injury surveillance systems keep a record of all head/neck/helmet impacts irrespective of whether or not they meet the threshold for a concussion diagnosis. That is, any impact to the head or neck region which would prompt the medical staff to ask the player whether they had suffered any symptoms (ie, generate medical attention based on the observed event rather than any symptoms), even if the player claims to have not suffered any injury or symptoms.

Injuries per number of player hours is, in theory, most accurate, but its relevance has been lessened by the rise of T20 cricket. $^{4}$ A day in which a T20 game is scheduled generating the same number of injuries as a day in which a 50-over match is scheduled would appear to have an injury incidence that was 2.5 times higher, because there were fewer overs bowled. Therefore, a unit of injuries (numerator) over player days (denominator, considering 11 players in action per day) could be used, which is simpler and more representative of actual risk (in a given time period). ${ }^{4}$ This is analogous to comparing injury rates of various Track athletic events in a per event rate $(100 \mathrm{~m}=$ one event; Marathon=one event) rather than a rate per hour of competition participation, which would make sprint events appear to be far riskier than distance events.

There are other circumstances specific to cricket where standard definitions are problematic. Within the realm of T20 cricket, some countries have moved to a 'franchise' model of T20 teams which are contractually and operationally distinct from the traditional domestic teams, whereas other countries still combine contracts to play T20 and longer forms of cricket for a single team. It would be inappropriate to state that T20 team cohorts should be either distinct from or combined with longer form surveillance cohorts, as the situation in different countries should lead to a different approach.

A somewhat unique cricket problem when assessing availability to play is the requirement of all players to be able to bat, but only some players need to bowl. The player who is fit to bat but not fit to bowl may be available for selection if their batting ability warrants, but not if selection would rely on also being fit to bowl. For some players who are 'part-time' bowlers but are unfit to bowl, it can be difficult and somewhat subjective to determine if they are missing a game whether this is truly due to injury or selector preference. This is particularly the case where a player continues to play as a batsman only and remains unfit to bowl. A guideline from these definitions is that this would equate to being unavailable through injury for a 'regular bowler', but we concede that these determinations may need to be made on a case-by-case basis. The difficulty is increased if a player is available to bowl but with workload limits recommended by medical staff that effectively result in non-selection in the longer forms of the game.

This complexity of role in cricket has led to our recommendation that injury surveillance systems avoid 'training time-loss' definitions, as many players regularly train in partial fashion. This would render the concept of a 'missed training session' to be too difficult to determine on too many occasions. For a 'general time-loss' injury, we have opted to recommend a daily binary status of 'considered fit to play a cricket match/not fit to play a cricket match' with the role and type of match necessarily being player-specific (ie, what type of match and in what role would a player be expected to be next fit for?).

In conclusion, these new consensus definitions are presented to offer greater flexibility to researchers at choosing methods which suit their study type and encouraging greater rigour in reporting to allow comparison between studies within cricket, and between sports. 


\section{Author affiliations}

${ }^{1}$ School of Public Health, University of Sydney, Sydney, Australia

${ }^{2}$ Cricket Australia, National Cricket Centre, Brisbane, Australia

${ }^{3}$ Cardiff School of Sport, Cardiff Metropolitan University, Cardiff, Wales, UK

${ }^{4}$ Department of Physiotherapy, Faculty of Health Sciences, University of the Witwatersrand, Johannesburg, Gauteng, South Africa

${ }^{5}$ Department of Orthopaedics, Post Graduate Institute of Medical Education and Research, Chandigarh, India

${ }^{6}$ Cricket South Africa, Cape Town, South Africa

${ }^{7}$ Exercise Science Camp, Sports Medicine, University of Cape Town, Cape Town,

South Africa

${ }^{8}$ England and Wales Cricket Board, National Cricket Performance Centre, Loughborough, UK

${ }^{9}$ Sports Medicine, University of the West Indies, Kingston, Jamaica

${ }^{10}$ New Zealand Cricket, High Performance Centre, Lincoln, New Zealand

${ }^{11}$ Section of Sports Medicine, Faculty of Health Sciences, University of Pretoria, Johannesburg, South Africa

${ }^{12}$ Department of Emergency Medicine, Faculty of Health Sciences, University of the Witwatersrand, Johannesburg, South Africa

${ }^{13}$ Sri Ramachandra University, Chennai, Tamil Nadu, India

${ }^{14}$ West Indies Cricket, St John's, Antigua and Barbuda

${ }^{15}$ Bangladesh Cricket Board, Sher-e- Bangla National Cricket Stadium, Dhaka, Bangladesh

${ }^{16}$ Sports Medicine Department, National Cricket Academy, Pakistan Cricket Board, Lahore, Pakistan

${ }^{17}$ National Cricket Academy, Cricket Scotland, Edinburgh, UK

${ }^{18}$ Sri Lanka Cricket, Colombo, Sri Lanka

${ }^{19}$ Zimbabwe Cricket, Harare, Zimbabwe

${ }^{20}$ Cricket Ireland, Dublin, Ireland

${ }^{21}$ Nottingham University Hospitals Trust, Centre for Sports Medicine, Nottingham, UK

${ }^{22}$ Nelson Mandela Metropolitan University, Port Elizabeth, South Africa

${ }^{23}$ Australian Centre for Research into Injury in Sport and its Prevention, Federation University Australia, Ballarat, Victoria, Australia

Twitter John Orchard @DrJohnOrchard, Craig Ranson @craigarxl, Isabel Moore @|zzyMoorePhD, Jon Patricios @jonpatricios, Nicholas Peirce @peirce123 and Caroline Finch, @CarolineFinch

Competing interests $\mathrm{JO}$ (and other authors affiliated with various national cricket boards and associations) declare an interest in being paid either salary or consultancies to provide services for the various associations that they are affiliated with. However, no author was funded or paid specifically to directly work on this paper or project.

Provenance and peer review Not commissioned; externally peer reviewed.

\section{REFERENCES}

1 Orchard JW, Newman D, Stretch R, et al. Methods for injury surveillance in international cricket. Br J Sports Med 2005;39:e22.

2 Frost WL, Chalmers DJ. Injury in elite New Zealand cricketers 2002-2008: descriptive epidemiology. Br I Sports Med 2014;4:1002-7.

3 Mansingh A, Harper L, Headley S, et al. Injuries in West Indies Cricket 2003-2004. Br J Sports Med 2006;40:119-23.
4 Orchard J, James T, Kountouris A, et al. Changes to injury profile (and recommended cricket injury definitions) based on the increased frequency of Twenty20 cricket matches. Open Access I Sports Med 2010;1:63-76.

5 Orchard JW, James T, Portus MR. Injuries to elite male cricketers in Australia over a 10-year period. J Sci Med Sport 2006;9:459-67.

6 Ranson C, Hurley R, Rugless L, et al. International cricket injury surveillance: a report of five teams competing in the ICC Cricket World Cup 2011. Br J Sports Med 2013;47:637-43.

7 Hodgson L, Gissane C, Gabbett TJ, et al. For debate: consensus injury definitions in team sports should focus on encompassing all injuries. Clin I Sports Med 2007; 17:188-91.

8 Mitchell R, Hayen A. Defining a cricket injury. J Sci Med Sport 2005;8:357-8; author reply 358-9.

9 Fuller CW, Ekstrand J, Junge A, et al. Consensus statement on injury definitions and data collection procedures in studies of football (soccer) injuries. Br I Sports Med 2006;40:193-201.

10 Fuller CW, Molloy MG, Bagate $C$, et al. Consensus statement on injury definitions and data collection procedures for studies of injuries in rugby union. $\mathrm{Br} /$ Sports Med 2007:41:328-31.

11 Orchard J, Hoskins W. For debate: consensus injury definitions in team sports should focus on missed playing time. Clin I Sports Med 2007;17:192-6.

12 Timpka T, Jacobsson J, Bickenbach J, et al. What is a sports injury? Sports Med 2014;44:423-8.

13 Clarsen B, Bahr R. Matching the choice of injury/illness definition to study setting, purpose and design: one size does not fit all! Br I Sports Med 2014;48:510-12.

14 Stretch R. Injuries to South African cricketers playing at first-class level. S Afr J Sports Med 1989:4:3-20.

15 Stretch RA. The incidence and nature of epidemiological injuries to elite South African cricket players. S Afr Med J 2001;91:336-9.

16 Stretch RA. Cricket injuries: a longitudinal study of the nature of injuries to South African cricketers. Br I Sports Med 2003;37:250-3; discussion 253.

17 Stretch RA. The incidence and nature of injuries in first-league and provincial cricketers. S Afr Med J 1993;83:339-42.

18 Finch CF, Cook J. Categorising sports injuries in epidemiological studies: the subsequent injury categorisation (SIC) model to address multiple, recurrent and exacerbation of injuries. Br J Sports Med 2014;48:1276-80.

19 Orchard J, Newman D, Stretch R, et al. Methods for injury surveillance in international cricket. N Z J Sports Med 2004:32:90-9.

20 Orchard J, Newman D, Stretch R, et al. Methods for injury surveillance in international cricket. J Sci Med Sport 2005;8:1-14.

21 Orchard J, Newman D, Stretch R, et al. Methods for injury surveillance in international cricket. S Afr I Sports Med 2005;17:18-28.

22 Orchard JW, Blanch P, Paoloni J, et al. Fast bowling match workloads over 5-26 days and risk of injury in the following month. I Sci Med Sport 2015:18:26-30.

23 Orchard JW, James T, Portus M, et al. Fast bowlers in cricket demonstrate up to 3- to 4-week delay between high workloads and increased risk of injury. Am J Sports Med 2009;37:1186-92.

24 Liberati A, Altman DG, Tetzlaff J, et al. The PRISMA statement for reporting systematic reviews and meta-analyses of studies that evaluate healthcare interventions: explanation and elaboration. BMJ 2009;339:b2700.

25 von Elm E, Altman DG, Egger M, et al., STROBE Initiative. Strengthening the Reporting of Observational Studies in Epidemiology (STROBE) statement: guidelines for reporting observational studies. BMJ 2007;335:806-8. 